\title{
Effect of finite computational domain on turbulence scaling law in both physical and spectral spaces
}

\author{
Thomas Y. Hou and Xiao-Hui Wu \\ Applied Mathematics, California Institute of Technology, Pasadena, California 91125 \\ Shiyi Chen \\ Center for Nonlinear Studies, Los Alamos National Laboratory, Los Alamos, New Mexico 87545 \\ Ye Zhou \\ IBM Research Division, T. J. Watson Research Center, Yorktown Heights, New York 10598 \\ and Institute for Computer Applications in Science and Engineering, NASA Langley Research Center, Hampton, Virginia 23681
}

(Received 22 December 1997; revised manuscript received 3 August 1998)

\begin{abstract}
The well-known translation between the power law of the energy spectrum and that of the correlation function or the second order structure function has been widely used in analyzing random data. Here, we show that the translation is valid only in proper scaling regimes. The regimes of valid translation are different for the correlation function and the structure function. Indeed, they do not overlap. Furthermore, in practice, the power laws exist only for a finite range of scales. We show that this finite range makes the translation inexact even in the proper scaling regime. The error depends on the scaling exponent. The current findings are applicable to data analysis in fluid turbulence and other stochastic systems. [S1063-651X(98)13911-9]

PACS number(s): 47.27.-i
\end{abstract}

\section{INTRODUCTION}

A fundamental question in turbulence research is the scaling laws of various physical quantities in the so-called inertial range, where those quantities usually scale as power laws. The scaling exponents are the focus of study. A famous example is Kolmogorov's $-\frac{5}{3}$ law for turbulence energy in the inertial range. This scaling behavior can be observed both in the physical space from the structure functions and in the spectral space from the energy spectrum. It is a common practice to relate power laws in the spectral space with those in the physical space as follows: given a homogeneous random field with an energy spectrum $E(k) \sim k^{-n}$, its correlation function $C(r)$ scales as $\sim r^{n-1}$, where $k$ is the wave number in the spectral space and $r$ is distance in the physical space. A similar relation holds for the second order structure function: $S(r) \sim r^{n-1}$.

Mathematically, the above translations hold only when $E(k)$ follows a pure power law that extends to $k=0$ and $\infty$ and with proper scaling exponents. These restrictions are not met by many physical problems, including turbulence. In particular, the power law of $E(k)$ may exist only in a finite "inertial range" : $k_{0} \leqslant k \leqslant k_{1}$, where $k_{0}$ and $k_{1}$ are the large scale cutoff and the dissipation scale cutoff, respectively. Since the wave number $k$ corresponds to a characteristic length scale $l=2 \pi / k$, the corresponding physical scale in the inertial range is given by $\left[r_{1}, r_{0}\right]$, where $r_{i}=2 \pi / k_{i}(i$ $=0,1)$. A long scaling range, namely, $k_{1} \gg k_{0}$ or $r_{0} \gg r_{1}$, is usually required for the study of the physics in that range. This is achieved, e.g., by high Reynolds number turbulent flows. In this case, the general practice is to ignore the effect of the finite scaling range.

In this paper we show that the finite scaling range does have important effects. More specifically, we study in detail the translation of power laws from spectral space to physical space in three dimensions (3D). We find that due to the finite power law range of $E(k), C(r)$ and $S(r)$ only approximately scale as a power law for $r_{1} \ll r \ll r_{0}$. Thus the power law range in the physical space is much shorter than that of the corresponding power law given in the spectral space. This phenomenon was mentioned in [1], page 62, but it has not been carefully analyzed. We show how the error of the power law approximation to $C(r)$ and $S(r)$ depends on the cutoff wave numbers, $k_{0}$ and $k_{1}$, as well as the scaling exponent $n$. The latter is of crucial importance. In fact, if $n$ is outside the proper ranges, one may obtain scaling exponents of $C(r)$ and $S(r)$ independent of $n$.

\section{PURE POWER LAW}

Let $u(\mathbf{x})$ be a random scalar field. Its Fourier representation is

$$
u(\mathbf{x})=\int_{R^{d}} \hat{u}(\mathbf{k}) e^{i \mathbf{k} \cdot \mathbf{x}} d \mathbf{k} .
$$

The correlation function and second order structure function are defined as $C\left(\mathbf{x}, \mathbf{x}^{\prime}\right)=\left\langle u(\mathbf{x}) u\left(\mathbf{x}^{\prime}\right)\right\rangle ; \quad S\left(\mathbf{x}, \mathbf{x}^{\prime}\right)=\langle| u(\mathbf{x})$ $\left.-\left.u\left(\mathbf{x}^{\prime}\right)\right|^{2}\right\rangle$, where \langle\rangle denotes an ensemble average. Assuming homogeneity: $\left\langle\hat{u}(\mathbf{k}) \hat{u}\left(\mathbf{k}^{\prime}\right)\right\rangle=Q(\mathbf{k}) \delta\left(\mathbf{k}+\mathbf{k}^{\prime}\right)$, we have that $C$ and $S$ are functions of $\mathbf{r}=\mathbf{x}-\mathbf{x}^{\prime}$ :

$$
\begin{gathered}
C(\mathbf{r})=\int_{R^{d}} Q(\mathbf{k}) e^{i \mathbf{k} \cdot \mathbf{r}} d \mathbf{k}, \\
S(\mathbf{r})=2 \int_{R^{d}} Q(\mathbf{k})\left(1-e^{i \mathbf{k} \cdot \mathbf{r}}\right) d \mathbf{k} .
\end{gathered}
$$

If we further assume $Q(\mathbf{k})=Q(k)(k=|\mathbf{k}|)$, then $C$ and $S$ are functions of $r=|\mathbf{r}|$. The energy spectrum $E(k)$ is given by $E(k)=S_{d} Q(k) k^{d-1}$, where $S_{d}=1,2 \pi$, and $4 \pi$ in one, two, and three dimensions, respectively. In $3 \mathrm{D}$, we have 


$$
\begin{gathered}
C(r)=\int_{0}^{\infty} E(k) \frac{\sin k r}{k r} d k, \\
S(r)=2 \int_{0}^{\infty} E(k)\left(1-\frac{\sin k r}{k r}\right) d k .
\end{gathered}
$$

Similar expressions can be obtained in general $d$-dimensional space, see, e.g., [2]. In the following, we use Eqs. (1) and (2) to demonstrate the relation between the power law scalings in the spectral and physical spaces. They can also be obtained for $d \neq 3$ in a similar fashion. It should be noted that the assumptions used in deriving Eqs. (1) and (2) are sufficient but not necessary. All derivations below use only Eqs. (1) and (2). Thus the results are applicable to any random field that satisfies Eqs. (1) and (2). In particular, it can be a vector field. When $E(k)=k^{-n}$ for $0<k<\infty$, we have a pure power law in the spectral space. We now derive the corresponding power law in the physical space. Letting $\rho=k r$, from Eq. (1) we have $C(r)=C_{0} r^{n-1}$, where $C_{0}$ is given by an improper integral

$$
C_{0}=\int_{0}^{\infty} \rho^{-(n+1)} \sin \rho d \rho,
$$

which exists for $-1<n<1$. Note that $n<1$ and $n>-1$ ensure the convergence of $C_{0}$ at $\rho=0$ and $\rho=\infty$, respectively. The value of $C_{0}$ can be found in [3]:

$$
C_{0}=\left\{\begin{array}{l}
\Gamma(-n) \sin (-n \pi / 2), \quad 0<n<1 \\
\pi / 2, \quad n=0 \\
\Gamma(1-n) \cos [(1-n) \pi], \quad-1<n<0 .
\end{array}\right.
$$

Similarly, we have $S(r)=S_{0} r^{n-1}$ with

$$
S_{0}=2 \int_{0}^{\infty} \rho^{-n}\left(1-\frac{\sin \rho}{\rho}\right) d \rho
$$

Unlike the case for $C(r)$, the improper integral $S_{0}$ exists only for $1<n<3$. We note that the ranges of $n$ in which $C_{0}$ and $S_{0}$ exist are different and do not overlap. Therefore in order to obtain the power law in the physical space one should choose the correlation function or the structure function according to the scaling exponent $n$.

\section{TRUNCATED POWER LAW}

In practice, $E(k)$ does not appear as a pure power law; it scales as a power law only in a certain range of $k$. Thus the integrals in Eqs. (3) and (5) contain no singularities and convergence is not a problem. However, we see below that the scaling behaviors of $C(r)$ and $S(r)$ are still dictated by the above convergence conditions for Eqs. (3) and (5), respectively.

In this section we assume $E(k)=k^{-n}$ in the interval $\left[k_{0}, k_{1}\right]\left(k_{1} \gg k_{0}\right)$ and $E(k) \equiv 0$ outside the interval. But the results presented in this paper should be valid also if $E(k)$ has a proper cutoff outside the interval. Then in general $C(r)=A(r) r^{n-1}$ and $S(r)=B(r) r^{n-1}$, where

$$
\begin{gathered}
A(r)=\int_{k_{0} r}^{k_{1} r} \rho^{-(n+1)} \sin \rho d \rho, \\
B(r)=2 \int_{k_{0} r}^{k_{1} r} \rho^{-n}\left(1-\frac{\sin \rho}{\rho}\right) d \rho .
\end{gathered}
$$

The fact that $A$ and $B$ are functions of $r$ indicates that $C(r)$ and $S(r)$ no longer follow a single power law. On the other hand, if $k_{0} r \ll 1$ and $k_{1} r \gg 1$, then $A \approx C_{0}$ and $B \approx S_{0}$, provided that $n \in(-1,1)$ and $n \in(1,3)$, respectively. In this case, $C(r)$ and $S(r)$ are approximately power laws with exponent $n-1$.

In the following, we analyze the effect of finite inertial range of $E(k)$ and that of $n$ on the scaling of correlation and structure functions. The main idea is to obtain asymptotic expansions for $C(r)$ and $S(r)$ in terms of $k_{0} r(\ll 1)$ and $k_{1} r(\gg 1)$. We would like to stress that $r$ need not be very small to achieve the expansions. In fact, $r$ is strictly in the physical space inertial range, i.e., $r_{1} \ll r \ll r_{0}$.

To fix the notation, throughout the paper, $A_{i}$ and $\alpha_{i}$ denote generic constants. These constants depend on $n$ and the dimension of space but independent of $r$. In addition, $A_{i}$ is independent of $k_{0}$ and $k_{1}$.

\section{A. A useful convergent expansion}

First let us provide a result which will be frequently used below. We consider the expansion of

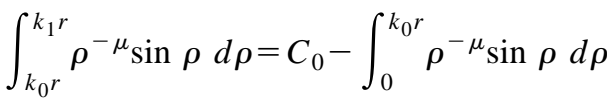

$$
\begin{aligned}
& -\int_{k_{1} r}^{\infty} \rho^{-\mu} \sin \rho d \rho,
\end{aligned}
$$

where $0<\mu<2$. Denote the first and second integrals on the right hand side by $I_{1}$ and $I_{2}$. Using the Taylor expansion of $\sin \rho$ for $I_{1}$ and integration by parts for $I_{2}$, we obtain

$$
\begin{gathered}
I_{1}=\frac{\left(k_{0} r\right)^{2-\mu}}{2-\mu}+O\left(\left(k_{0} r\right)^{4-\mu}\right), \\
I_{2}=\left(k_{1} r\right)^{-\mu} \cos k_{1} r+O\left(\left(k_{1} r\right)^{-(1+\mu)}\right),
\end{gathered}
$$

respectively. Therefore to the leading orders we have

$$
\int_{k_{0} r}^{k_{1} r} \rho^{-\mu} \sin \rho d \rho \approx C_{0}-\frac{\left(k_{0} r\right)^{2-\mu}}{2-\mu}-\left(k_{1} r\right)^{-\mu} \cos \left(k_{1} r\right) .
$$

This expansion converges to $C_{0}$ as $k_{0} r \rightarrow 0$ and $k_{1} r \rightarrow \infty$. The convergence becomes slow when $\mu$ is close to 2 or 0 .

In the following, we expand $A(r)$ and $B(r)$. When $n$ is in the proper ranges, Eq. (8) gives the desired result. Otherwise, we may repeatedly apply integration by parts to $A(r)$ and $B(r)$ until the exponent of $\rho$ in the remaining integral falls into the range $(0,2)$. By Eq. (8), this remaining integral gives the constant term in $A(r)$ and $B(r)$, hence the $A_{0} r^{n-1}$ term in $C(r)$ and $S(r)$. However, this term may be dominated by the terms generated from the integration by parts, which are functions of $r$. In these cases, the $A_{0} r^{n-1}$ term is retained in 
the expansions for the purpose of comparison while the other two terms in Eq. (8) are neglected. It should be noted that the repeated integration by parts just mentioned is always possible if $n$ is not an integer. For simplicity we assume this is true in the expansions below.

\section{B. Power law approximation for correlation function}

Case $-1<n<1$. Since $0<1+n<2$, it follows immediately from Eq. (8) that

$$
C(r) \approx r^{n-1}\left[C_{0}+A_{1}\left(k_{0} r\right)^{1-n}-\cos \left(k_{1} r\right)\left(k_{1} r\right)^{-(n+1)}\right] .
$$

Therefore the power law of $C(r)$ is only an approximate one. In practice, one obtains the scaling exponent of $C(r)$ by fitting it with a power function

$$
f_{1}(r)=\alpha_{0} r^{m}
$$

where $\alpha_{0}$ and $m$ are constants to be determined by a least squares procedure. Equation (9) indicates that in order to obtain an accurate exponent one should choose $C(r)$ in an $r$ range such that $\left(k_{0} r\right)^{1-n} \ll 1$ and $\left(k_{1} r\right)^{-(n+1)} \ll 1$. Note that the proper choice is determined by the truncation modes as well as the exponent. On the other hand, because the leading order error due to the truncation at $k_{0}$ cancels with $r^{n-1}$, we may better fit $C(r)$ using a function of the form

$$
f_{2}(r)=\alpha_{0} r^{m}+\alpha_{1}
$$

It should be noted that the least squares fit now involves solving a nonlinear system of equations.

Case $n>1$. By Taylor expansions of $\sin \rho$ and integration by parts, we obtain from Eq. (8)

$$
C(r) \approx A_{0} r^{n-1}+A_{1} k_{0}^{1-n}+A_{2} k_{0}^{3-n} r^{2} .
$$

This expansion indicates that the error mainly comes from the low-mode truncation and the error diverges as $k_{0} \rightarrow 0$. Note that the last term is relatively small if $n<3$. In this case, we can use Eq. (10) to extract the scaling exponent from $C(r)$. But if $n>3$, the last term dominates the first term since $A_{2}\left(k_{0} r\right)^{3-n} \gg A_{0}$. Thus, using Eq. (10) one may obtain $m \approx 2$ regardless of the value of $n$. A simple fix seems to be including the $r^{2}$ term in the fitting function, i.e.,

$$
f_{3}(r)=\alpha_{0} r^{m}+\alpha_{1}+\alpha_{2} r^{2} .
$$

However, this does not solve the whole problem because the terms neglected in the expansion (11) may dominate the first term if $n$ is large. For example, if $n>5$, a $k_{0}^{5-n} r^{4}$ term in the expansion becomes larger than the first and hence Eq. (12) does not work.

Case $n<-1$.

$$
C(r) \approx A_{0} r^{n-1}+A_{1} k_{1}^{-(n+1)} r^{-2} \cos \left(k_{1} r\right),
$$

fixed $r, C(r) \rightarrow \infty$ as $k_{1} \rightarrow \infty$ due to the second term. This term, while containing fast oscillations, $\cos \left(k_{1} r\right)$, decays slower than $r^{n-1}$. Thus it is dominating. We may replace $r^{2}$ in Eq. (12) by $r^{-2}$ to reflect the asymptotics; however, this modification is not helpful because of the rapid oscillations in the coefficient.

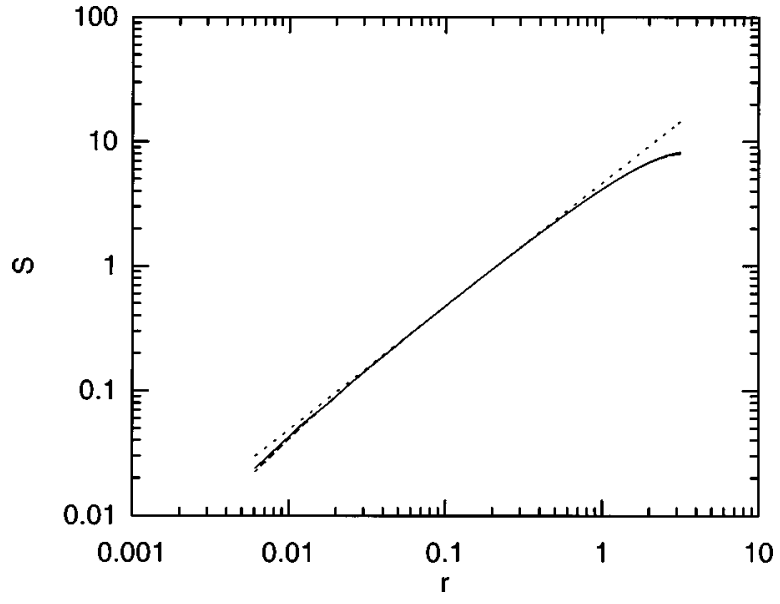

FIG. 1. Log-log plot of $S(r)$ for $n=2$. Solid line: $S(r)$; dashed line: fitted curve using $f_{3}(r)$ with $m=1$; dotted line: fitted curve using $f_{1}(r)$.

\section{Power law approximation for structure function}

The structure function $S(r)$ can be analyzed using the same approach as above. In particular, we observe

$$
\begin{aligned}
\int \rho^{-n}\left(1-\frac{\sin \rho}{\rho}\right) d \rho= & \int \rho^{-(1+n)}(\rho-\sin \rho) d \rho \\
= & -\frac{\rho-\sin \rho}{n \rho^{n}}-\frac{1-\cos \rho}{n(n-1) \rho^{n-1}} \\
& -\frac{1}{n(n-1)} \int \rho^{1-n} \sin \rho d \rho .
\end{aligned}
$$

Thus the results for $C(r)$ can be applied directly to the last integral.

Case $1<n<3$. It is straightforward to derive

$$
\begin{aligned}
S(r) \approx & r^{n-1}\left[A_{0}+A_{1}\left(k_{0} r\right)^{3-n}+A_{2}\left(k_{1} r\right)^{1-n}\right. \\
& \left.+A_{3}\left(k_{1} r\right)^{-n} \sin \left(k_{1} r\right)\right] .
\end{aligned}
$$

Note that the last term can be neglected because $k_{1} r \gg 1$. The terms with $A_{1}$ and $A_{2}$ are small if $n$ is not close to 3 and 1 , respectively. In this case, one can directly extract the exponent by fitting $S(r)$ with $f_{1}(r)$. However, $f_{3}(r)$ is a better fitting function which includes the effect of both terms. This is demonstrated in Fig. 1, where $S(r)$ of a 2D random field with $E(k)=k^{-2}(k \in[1,512])$ is plotted. Fast Fourier transform (FFT) is used in computing $S(r)$ and generating the random field in the $(2 \pi)^{2}$ domain on a $1024^{2}$ lattice. The dotted line is obtained by using $f_{1}$. It has a slope of 0.99 , quite close to the exact value, 1 . When using $f_{3}$, to avoid solving the nonlinear system of equations, we let $m=1$ and compute $\alpha_{i}$. We have $\alpha_{0}=4.92, \alpha_{1}=-0.00751$, and $\alpha_{2}$ $=-0.757$, indicating that $r^{n-1}$ scaling dominates. In addition, our tests show that the fitting results are sensitive to the data used. The result reported here is obtained from fitting $S(r)$ in the $r$ interval $[0.098,0.196]$, which satisfies the condition $r_{1} \ll r \ll r_{0}$. Violating this condition renders an inaccurate fitted scaling exponent. Furthermore, we note that the Kolmogorov law for homogeneous turbulence, having $n$ $=\frac{5}{3}$, belongs to this case. 


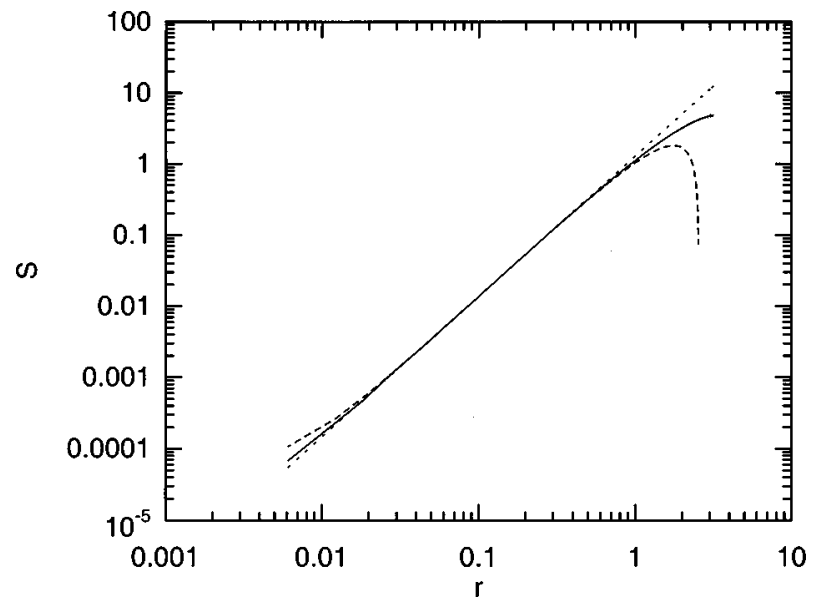

FIG. 2. Log-log plot of $S(r)$ for $n=4.5$. Solid line: $S(r)$; dashed line: fitted curve using $f_{3}(r)$ with $m=3.5$; dotted line: fitted curve using $f_{1}(r)$.

Case $n>3$. We have

$$
S(r) \approx A_{0} r^{n-1}+A_{1} k_{0}^{3-n} r^{2} .
$$

Equation (13) differs from Eq. (11) only by a constant term. The above test is performed again here with $n=4.5$. The results are shown in Fig. 2. The slope of the dotted line (fitting with $f_{1}$ ) is 1.98 , close to 2 instead of $n-1=3.5$ as predicated by Eq. (13). Letting $m=3.5$ in $f_{3}$, we find $\alpha_{0}=$ $-0.338, \alpha_{1}=5.69 \times 10^{-5}$, and $\alpha_{2}=1.38$. Thus the $r^{2}$ term is indeed larger. Our tests show that increasing $n$ makes $\alpha_{0} / \alpha_{2}$ smaller and hence more dominant $r^{2}$ scaling in $S(r)$. It is also seen from the figure that $f_{3}$ does not fit better than $f_{1}$ due to large $n$.

Case $n<1$. The leading orders of $S(r)$ are

$$
S(r) \approx A_{0} r^{n-1}+A_{1} k_{1}^{1-n}+A_{2} k_{1}^{-n} r^{-1} \sin \left(k_{1} r\right) .
$$

Note that for fixed $r, S(r) \rightarrow \infty$ as $k_{1} \rightarrow \infty$. $I_{\mu}$ here contains only $I_{1}$. Furthermore, $2-\mu=1-n, I_{\mu}$ canceling with $r^{n-1}$ gives a constant term. When $n>0$, Eq. (10) can be used since the last term is small. However, when $n<0$, we have a situation similar to that of $C(r)$ when $n<-1$.

\section{POWER LAWS WITH SMOOTH CUTOFF}

In most real physical processes, the power laws are not truncated sharply but with smooth cutoff functions. It is straightforward to include the effect of these functions in the above derivation. Indeed, from the derivation of Eq. (8) we

[1] U. Frisch, Turbulence (Cambridge University Press, Cambridge, England, 1995).

[2] I. S. Reed, P. C. Lee, and T. K. Truong, IEEE Trans. Inf. Theory 41, 1439 (1995). see that the contributions of the smooth cutoff functions are rather small under the assumptions $k_{0} r \ll 1$ and $k_{1} \gg 1$. This is because the cutoff functions are regular at $k=0$ and usually much less singular as $k \rightarrow \infty$ than the power law itself. Therefore the results obtained above for the inertial range also hold for the cases with smooth cutoffs. On the other hand, the cutoff functions should have a strong effect outside the inertial range. For example, they would change the bending at the two ends of the power law in the physical space in Fig. 1.

\section{CONCLUDING REMARKS}

In this paper we have studied the translation between the power law of the energy spectrum and that of the correlation function or the second order structure function. We have obtained the following four conclusions.

(1) Power laws in spectral and physical spaces have simple correspondence only for $n$ in proper ranges, i.e., $(-1,1)$ and $(1,3)$, respectively, for the correlation and structure functions. The effect of finite power law range in the spectral space results in a much shorter power law range in the physical space.

(2) Based on the asymptotic expansions, a fitting function $f_{3}$ is proposed for better recovery of $n$ from $S(r)$ and $C(r)$. For a given value of $n$, we recommend using either $S(r)$ $(1<n<3)$ or $C(r)(-1<n<1)$ for studying the corresponding power law in the physical space.

(3) When $n$ is outside the proper ranges, the correlation and structure functions are generally dominated by some functions of $r$ that are independent of $n$. Thus recovering $n$ is very difficult, if not impossible.

(4) Following the analysis outlined above, one finds that the translation of a power law from the physical space to the spectral space suffers similar problems.

A fundamental question relevant to the above observations is, which space in the power law of a physical process, such as turbulence, has a wider range of applicability intrinsically? For homogeneous turbulence in a periodic box, various numerical studies seem to indicate that the spectral space is more "intrinsic" (in the sense that the power law range is longer). However, a general answer to the question is beyond the scope of this paper.

\section{ACKNOWLEDGMENT}

Y.Z. was partially supported by ICASE through NASA Contract No. NAS1-19480.

[3] I. S. Gradshteyn and I. M. Ryzhik, in Table of Integrals, Series, and Products, edited by A. Jeffrey (Academic, New York, 1994). 